\title{
O uso da aprendizagem baseada em problemas na Odontologia: uma revisão crítica da literatura
}

\author{
Juliana Schaia Rocha*; Gisele Fernandes Dias*; Nara Hellen Campanha**; Márcia Helena Baldani**
}

\section{RESUMO}

A sociedade contemporânea impõe desafios constantes aos setores da saúde e da educação, alicerçada em revisões e reformulações permanentes para formação de recursos humanos. Este artigo promove uma revisão crítica da literatura a respeito da aprendizagem baseada em problemas (Problem Based Learnig - PBL) direcionada ao ensino da Odontologia. As bases de dados consultadas foram Medline (via EBSCO), SCIELO, BBO e LILACS (via BVS). As palavras-chave foram definidas baseadas em artigos previamente selecionados. Esta metodologia se apresenta como uma alternativa para formação e educação em Odontologia. É considerada uma ferramenta de trabalho para professores que buscam desenvolver em seus alunos capacidades cognitivas promotoras do desenvolvimento do raciocínio clínico integrado, com perfil direcionado à educação permanente.

Descritores: Aprendizagem Baseada em Problemas. Odontologia. Ensino.

\section{INTRODUÇÃO}

$\mathrm{O}$ ensino tradicional tem passado por uma série de questionamentos e mudanças
Doutoranda, Programa de Pós-Graduação em Odontologia, Universidade Estadual de Ponta Grossa ** Professora do Departamento de Odontologia da Universidade Estadual de Ponta Grossa

para atender às exigências das Diretrizes Curriculares Nacionais (DCN). Essas diretrizes definem a necessidade de um perfil generalista do cirurgião-dentista, com habilidades e competências a serem desenvolvidas. E, para isso, são necessárias a discussão e a construção de novos projetos pedagógicos nos cursos de Odontologia e mudanças curriculares ${ }^{1}$.

As habilidades gerais preconizadas pelas DCN são de atenção à saúde, tomada de decisão, comunicação, liderança, administração e gerenciamento, além de educação permanente. Para desenvolvê-las, o aluno deve ter diversas capacidades, dentre elas a de colher, observar e interpretar dados para a construção do diagnóstico; desenvolver o raciocínio lógico e a análise crítica na conduta clínica; propor e executar planos de tratamento adequados; realizar a promoção e a manutenção da saúde; comunicar-se com pacientes, com profissionais de saúde e com a comunidade em geral, dentro de preceitos éticos e legais; trabalhar em equipes interdisciplinares e atuar como agente de promoção de saúde; planejar e administrar 
serviços de saúde coletiva ${ }^{1}$.

O ensino tradicional na Odontologia resulta em dificuldades para o desenvolvimento destas habilidades no aluno. As dificuldades surgem devido ao fato de que os cursos possuem padrões curriculares fragmentados, cabendo ao aluno realizar a integração dos conteúdos ${ }^{2}$. Esse modelo possui aulas apresentadas para grandes grupos, onde o professor é detentor do conhecimento. Este é depositado ou transferido para o aluno ouvinte ${ }^{3}$, que participa do processo ensino-aprendizagem de forma passiva. Os currículos são estruturados em disciplinas básicas e específicas não integradas, que se tornam muitas vezes repetitivas ${ }^{4}$.

A Odontologia é uma ciência complexa e integrada, logo o processo educacional também deve seguir esse padrão. Para isso, têm-se estudado as metodologias ativas de ensino, que são conceituadas como "processos interativos de conhecimento, análises, estudos, pesquisas e decisões individuais ou coletivas, com a finalidade de encontrar soluções para um problema"5. Baseiam-se no conceito de que o que impulsiona a aprendizagem é a superação de desafios, resolução de problemas e construção do conhecimento a partir de experiências prévias ${ }^{6}$.

O professor deixa de ser um detentor e transmissor do conhecimento e passa a ser um facilitador/orientador nas pesquisas, reflexões e tomadas de decisão do aluno. Sendo assim, as metodologias ativas ensinam o aluno a aprender a aprender, através de experiências reais ou simuladas, favorecendo também a educação permanente ${ }^{67}$.

Dentre as metodologias ativas está a aprendizagem baseada em problemas (Problem Based Learning - PBL). Na PBL o ensino é centralizado no aluno, sendo ele o responsável pela busca do conhecimento de forma ativa. $\mathrm{O}$ ensino consiste na integração de conteúdos das várias áreas envolvidas, diferente do ensino tradicional que divide o conhecimento em disciplinas básicas e específicas. Essa metodologia tem como um dos objetivos desenvolver no aluno a capacidade de procurar soluções para problemas que surgem durante toda a sua vida profissional $^{8}$.

\section{Fundamentação teórica da metodologia PBL}

A PBL é um modelo pedagógico que se iniciou em 1969, na educação médica da Escola de Medicina da Universidade McMaster em Ontário, Canadá. Na década de 90 ela foi incluída nos cursos de medicina em todo o mundo e aprovado pela Federação Mundial de Educação Médica e da Organização Mundial de Saúde ${ }^{9}$. Sua introdução no ensino Odontológico iniciou muito mais tarde, em meados de 1990, com as escolas na Suécia, Austrália, EUA, Hong Kong, Irlanda e Reino Unido ${ }^{10}$.

A abordagem PBL se diferencia das outras metodologias ativas por constituir-se como o eixo principal do aprendizado técnicocientífico dentro de uma proposta curricular ${ }^{7}$, em que o ensino está centrado no aluno. Desta maneira, os alunos devem definir e estabelecer objetivos de aprendizagem necessários para desenvolver sua compreensão de determinado problema, além de uma busca ativa do conhecimento $^{11}$.

A PBL tem como objetivos principais assimilar novos conhecimentos a partir da integração de diferentes disciplinas, desenvolver uma abordagem para análise de situações clínicas e capacidade de avaliar seu próprio desempenho, além de desenvolver uma boa comunicação interpessoal e 
autonomia de aprendizado e favorecer a educação permanente ${ }^{12}$.

Esta pedagogia baseia-se em pequenos grupos de alunos trabalhando juntos na solução de um problema, sob orientação de facilitadores do corpo docente para alcançar os objetivos. O processo de aprendizagem ocorre da seguinte forma ${ }^{13}$ : 1) os alunos são apresentados a uma situação problema, simulada, que definirá as prioridades de conhecimento; 2) os alunos trabalham em pequenos grupos com um facilitador para esclarecer termos e conceitos, analisar e interpretar a situação e identificar os problemas; 3) os grupos geram hipóteses de trabalho sobre as causas e consequências dos problemas; 4) o grupo identifica questões decorrentes do problema (questões de aprendizagem) para um estudo auto-dirigido; 5) os alunos se reúnem para compartilhar as informações pesquisadas e tentar explicar e encontrar respostas para o problema. Essas informações serão determinantes para o diagnóstico e planejamento do tratamento. $\mathrm{O}$ facilitador também contribui com informações nesta etapa; 6) Após a solução do problema, deve ser realizada a avaliação das habilidades adquiridas pelo aluno durante o processo.

É importante destacar que o facilitador não atua como professor ou como um especialista no conteúdo, mas procura ajudar os alunos a trabalharem com o problema (caso) para obter o máximo benefício a partir de sua aprendizagem. Um exemplo de aplicação prática da metodologia PBL em Odontologia pode ser visto no estudo de Zheng et $\mathrm{al}^{14}$, no ensino de cirurgia bucal e maxilofacial. $\mathrm{O}$ primeiro tutorial traz a descrição de um caso com os principais pontos para a discussão. Os tutoriais seguintes trazem a resolução do caso passo a passo com os cenários para discussão em cada encontro. No último encontro são resumidos os pontoschaves de cada cenário e dada a literatura complementar sobre o assunto.

Diante do exposto, este trabalho tem como objetivo revisar a literatura sobre a aplicação da metodologia ativa "Aprendizagem Baseada em Problemas" na Odontologia e verificar os avanços já alcançados.

\section{MATERIAL E MÉTODOS}

Este estudo é uma revisão crítica de literatura, baseada em uma busca estratégica de publicações. As bases de dados utilizadas foram: Medline (via EBSCO), SCIELO, BBO e LILACS (via BVS). As palavras-chave foram definidas baseadas em artigos previamente selecionados, as quais foram: educação em Odontologia, Odontologia, aprendizagem baseada em problemas e PBL nos idiomas português, inglês e espanhol, limitados aos últimos 10 anos (2005-2015). A estratégia de busca utilizada em cada base está representada no quadro 1 .

Os critérios de inclusão utilizados foram estudos que explorassem o tema "Aprendizagem Baseada em Problemas na Odontologia". Foram excluídos aqueles estudos que 1) envolviam apenas cursos de outras áreas da saúde fora da Odontologia; 2) estudos não publicados, como tese $\mathrm{e}$ dissertações; 3) estudos com texto completo não disponível 4) não tinham como objetivo principal a PBL.

Após as buscas realizadas, foram excluídos os artigos duplicados. Em seguida, os artigos foram eliminados por título e resumo. Os resumos condizentes com os critérios adotados (citados no parágrafo anterior) foram selecionados e incluídos nesta revisão crítica. 


\section{RESULTADOS}

Foram recuperados 210 artigos, a partir das palavras-chave. Após a remoção das duplicações e da utilização dos critérios previamente definidos, restaram 25 artigos (figura 1).

A tabela 1 apresenta a relação dos artigos selecionados. Dos 25 artigos encontrados na literatura, 7 são de revisão de literatura, 5 comparam o ensino tradicional com a PBL, 12 mostram experiências da PBL na Odontologia e avaliação dos alunos e 1 discute uma metodologia para capacitação docente.

Os tópicos a seguir sintetizam os assuntos abordados nos artigos selecionados, na seguinte sequência: experiências na Odontologia, sucessos e desafios e ensino tradicional versus aprendizagem baseada em problemas.

Quadro 1. Estratégia de busca utilizada nas bases pesquisadas.

\begin{tabular}{|c|c|}
\hline \multicolumn{2}{|c|}{ Medline (via ebsco) } \\
\hline \#1 & \#2 \\
\hline $\begin{array}{l}\text { (TI "dental education" OR AB "dental education" OR } \\
\text { MH "dental education" OR TI dentistry OR AB } \\
\text { dentistry OR MH dentistry }\end{array}$ & $\begin{array}{l}\text { AB problem based learning OR TI problem based } \\
\text { learning OR MH problem based learning OR AB PBL } \\
\text { OR TI PBL }\end{array}$ \\
\hline \multicolumn{2}{|c|}{ \#1 AND \#2 } \\
\hline \multicolumn{2}{|c|}{ SCIELO* } \\
\hline \multicolumn{2}{|l|}{ \#1 } \\
\hline \multicolumn{2}{|c|}{ "aprendizagem baseada em problemas" OR "PBL" OR "problem-based learning" } \\
\hline \multicolumn{2}{|c|}{ LILACS e BBO (via BVS) } \\
\hline \#1 & $\# 2$ \\
\hline $\begin{array}{l}\text { tw:(educación en odontologia)) OR (tw:(dental } \\
\text { education)) OR (tw:(educação em odontologia)))) OR } \\
\text { (tw:(odontologia)) OR (tw:(dentistry)) }\end{array}$ & $\begin{array}{l}\text { tw:(problem-based learning)) OR (tw:(Aprendizaje } \\
\text { Basado em Problemas }\end{array}$ \\
\hline \multicolumn{2}{|c|}{ \#1 AND \#2 } \\
\hline
\end{tabular}

*Filtro: Ciências da Saúde

Figura 1. Fluxograma dos artigos do estudo, após critérios de inclusão e exclusão.

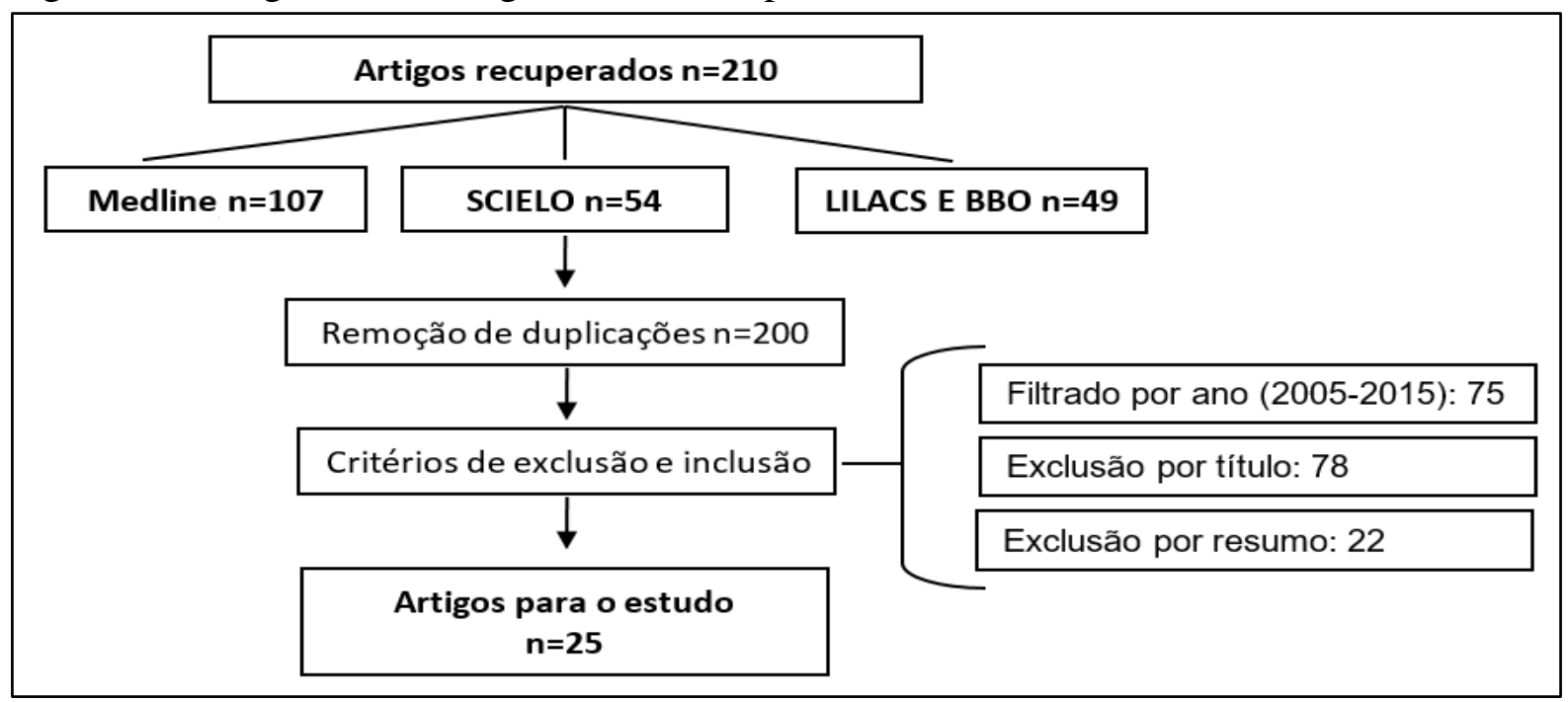


Tabela 1. Relação dos artigos mantidos após aplicados os critérios de inclusão e exclusão.

\section{Autor/ano}

Saunders e

Dejbakhsh $^{15}(2007)$ Tipo do estudo Capacitação docente à PBL

Thammasitboon et al. ${ }^{16}(2007)$

Comparação entre PBL e ensino tradicional

Estudo de caso da PBL

Huang et al. ${ }^{17}$ (2007)

Winning e Townsend Revisão de literatura

13 (2007)

Saliba et al. ${ }^{18}$ (2008) Revisão de literatura

Ajjawi et al. ${ }^{19}$ (2009) Estudo de caso da PBL

Ji et al. ${ }^{20}$ (2010)

Polyzois et al. ${ }^{21}$ (2010)

Whitney e Walton ${ }^{22}$

(2010)

Bearn e Chadwick ${ }^{23}$

(2010)

Haden et al. ${ }^{24}(2010)$
Comparação entre PBL e ensino tradicional

Comparação entre PBL e ensino tradicional

Estudo de caso da PBL

Estudo de caso da PBL

Estudo de caso da PBL

\section{Objetivos}

Apresentar uma metodologia de capacitação docente ao ensino PBL

Comparar o desempenho de graduados em PBL com graduados do ensino tradicional

Apresentar as mudanças de currículo da Escola de Estomatologia da Universidade Wuhan $-$

Apresentar uma revisão de literatura e uma observação in loco do método

Estudar as experiências de alunos de Odontologia que compartilham módulos da PBL com a medicina

Avaliar os efeitos de um curso ministrado em 2 grupos: ensino em PBL e tradicional Avaliar as diferenças entre graduados em PBL e o ensino tradicional a longo prazo Estudar as percepções de alunos e corpo docente após uma reforma curricular Estudar experiências de alunos de pósgraduação ensinados por meio da PBL

Avaliar currículos de escolas de Odontologia

\section{Conclusões}

A capacitação docente para a PBL é fundamental. O artigo apresenta uma proposta para capacitar facilitadores em 4 wokshops: Processo da PBL, Facilitação da Aprendizagem, Avaliação dos alunos e feedback e PBL no ambiente clínico.

O desempenho durante a pós-graduação dos alunos formados em PBL é semelhante ao dos formados com o ensino tradicional. Porém, os formados em PBL tem habilidades em aprendizagem independente, cooperação e comunicação.

Inovações curriculares estão nos passos iniciais na China. Ainda são necessários estudos para avaliar sua implementação e superação de desafios

Programas híbridos com a PBL bem planejados podem estimular o

desenvolvimento de habilidades e atributos para a vida profissional do aluno.

Traz reflexões para a implantação da metodologia PBL, sugerindo um currículo flexível, organizado em blocos temáticos, orientado para a solução de problemas, com integração de conteúdos básicos e clínicos, com equilíbrio teoria-prática, dinâmico, que atenda às mudanças no perfil epidemiológico da população.

Fatores contextuais podem impactar negativamente na experiência de alunos educados em PBL

O senso de responsabilidade com a profissão foi aprimorado em alunos educados com a PBL, quando comparados ao ensino tradicional

A PBL não melhorou a aprendizagem ao longo da vida. As variáveis demográficas e profissionais parecem ter um papel importante.

Embora a PBL funcione, foram identificados alguns pontos para a melhoria do curso.

Houve conflito de opiniões em relação à eficácia da PBL. Foi proposto um constructo para ajudar na compreensão da experiência da PBL.

A maioria dos currículos está em processo de renovação ou foram modificados nos últimos 2 anos. Houve um aumento no percentual de escolas com cursos interdisciplinares, mas nenhuma mudança no uso da PBL. 


\begin{tabular}{|c|c|c|c|}
\hline $\begin{array}{l}\text { Haghparast et al. } \\
\text { (2011) }\end{array}$ & Estudo de caso da PBL & $\begin{array}{l}\text { Comparar os objetivos alcançados na } \\
\text { aprendizagem entre } 4 \text { grupos ensinados em } \\
\text { PBL }\end{array}$ & $\begin{array}{l}\text { Embora haja uma variabilidade na aprendizagem dos grupos estudados, a } \\
\text { elaboração de questões norteadoras pode guiar a investigação de conceitos } \\
\text { importantes. }\end{array}$ \\
\hline Yiu et al. $^{26}(2011)$ & $\begin{array}{l}\text { Comparação entre PBL e } \\
\text { ensino tradicional }\end{array}$ & $\begin{array}{l}\text { Comparar um grupo de graduados em PBL } \\
\text { com um graduado no ensino tradicional }\end{array}$ & $\begin{array}{l}\text { Não houve diferenças no conhecimento clínico entre os alunos formados } \\
\text { na Universidade de Hong Kong com um currículo tradicional e PBL. }\end{array}$ \\
\hline Zheng et al. ${ }^{14}$ (2011) & Estudo de caso da PBL & $\begin{array}{l}\text { Estudo de um caso clínico em PBL em uma } \\
\text { turma de Odontologia }\end{array}$ & $\begin{array}{l}\text { Traz o passo a passo do ensino em PBL no ensino de cirurgia bucal e } \\
\text { maxilofacial. }\end{array}$ \\
\hline Pardamean $^{27}$ (2012) & Estudo de caso da PBL & $\begin{array}{l}\text { Estudo das habilidades de alunos educados } \\
\text { com a PBL }\end{array}$ & $\begin{array}{l}\text { Não houve diferenças na aprendizagem ao longo da vida entre alunos que } \\
\text { receberam o ensino tradicional e a PBL. }\end{array}$ \\
\hline Huang et al. ${ }^{28}(2013)$ & Revisão de Literatura & Revisão de estudos com a PBL na China & A metodologia PBL se apresentou como superior ao ensino tradicional. \\
\hline $\begin{array}{l}\text { Nadershahi et al. }{ }^{29} \\
\text { (2013) }\end{array}$ & Revisão de Literatura & Baseada na literatura médica & $\begin{array}{l}\text { A PBL tem sido uma das ferramentas promissoras para educadores } \\
\text { médicos e odontológicos }\end{array}$ \\
\hline Teodoro $^{30}(2014)$ & Estudo de caso da PBL & $\begin{array}{l}\text { Avaliar um caso da PBL em uma turma de } \\
\text { graduação }\end{array}$ & $\begin{array}{l}\text { A PBL facilita o desenvolvimento das habilidades do aluno, com um alto } \\
\text { nível de desempenho acadêmico e satisfação dos alunos. }\end{array}$ \\
\hline $\begin{array}{l}\text { Navazesh et al. }{ }^{31} \\
\text { (2014) }\end{array}$ & Estudo de caso da PBL & $\begin{array}{l}\text { Relato de caso da Faculdade de Ostrow de } \\
\text { Odontologia (OSDUSC) }\end{array}$ & $\begin{array}{l}\text { Através da reorganização e integração de conteúdo, foi condensado o } \\
\text { currículo tradicional de } 190 \text { para } 44 \text { cursos. Ainda está sendo aprimorada } \\
\text { a metodologia para se ter um currículo centrado no aluno. }\end{array}$ \\
\hline Bassir et al..$^{8}(2014)$ & Revisão de literatura & $\begin{array}{l}\text { Avaliar a eficácia do ensino em PBL } \\
\text { comparado ao tradicional }\end{array}$ & $\begin{array}{l}\text { Há um número muito limitado de estudos controlados e bem desenhados } \\
\text { que avaliam a eficácia da PBL na educação odontológica. }\end{array}$ \\
\hline $\begin{array}{l}\text { Moreno-López et al. }{ }^{32} \\
(2009)\end{array}$ & $\begin{array}{l}\text { Comparação entre PBL e } \\
\text { ensino tradicional }\end{array}$ & $\begin{array}{l}\text { Comparar um caso de PBL com o ensino } \\
\text { tradicional entre alunos de uma turma de } \\
\text { graduação }\end{array}$ & $\begin{array}{l}\text { Apesar dos resultados de aprendizagem avaliados serem ligeiramente } \\
\text { maiores em alunos PBL, não houve diferenças estatisticamente } \\
\text { significativas entre os grupos. }\end{array}$ \\
\hline $\begin{array}{l}\text { Bengmark et al. }{ }^{33} \\
(2012)\end{array}$ & Estudo de caso da PBL & $\begin{array}{l}\text { Avaliar como alunos graduados em uma } \\
\text { Universidade com ensino em PBL, } \\
\text { percebem sua formação e satisfação } \\
\text { profissional }\end{array}$ & $\begin{array}{l}\text { A educação em PBL foi percebida positivamente pelos formandos no } \\
\text { preparo para a vida profissional e melhoria da satisfação profissional. }\end{array}$ \\
\hline Rimal et al.34 (2015) & Estudo de caso da PBL & $\begin{array}{l}\text { Avaliar o conhecimento de alunos antes e } \\
\text { após um módulo de PBL }\end{array}$ & $\begin{array}{l}\text { PBL incentivou os alunos a usar métodos mais interativos de aprendizagem } \\
\text { que, possivelmente, irá torná-los eternos aprendizes. }\end{array}$ \\
\hline $\begin{array}{l}\text { Townsend e } \\
\text { Winning } \\
\text { Win (2011) }\end{array}$ & Revisão de literatura & Baseado na literatura médica & $\begin{array}{l}\text { A maior parte da investigação sobre PBL é relacionada à educação } \\
\text { médica e está sendo publicada em revistas de educação médica. }\end{array}$ \\
\hline $\begin{array}{l}\text { Townsend et al. }{ }^{35} \\
\text { (2012) }\end{array}$ & Revisão de literatura & $\begin{array}{l}\text { Apresentação um novo modelo conceitual } \\
\text { para pesquisa da PBL }\end{array}$ & $\begin{array}{l}\text { O modelo conceitual apresentado pode nos ajudar a avançar, tanto na futura } \\
\text { aplicação da PBL na educação odontológica, quanto em relação à } \\
\text { elaboração de novas questões de pesquisa. }\end{array}$ \\
\hline
\end{tabular}




\section{Experiências na Odontologia}

A inserção da PBL na Odontologia tem sido iniciada de forma gradativa nas faculdades de Odontologia. Em 2009 foram avaliados os currículos dos cursos de Odontologia do Canadá e dos EUA sobre o formato, avaliação e inovação do currículo. Quarenta e nove por cento dos entrevistados definiram seu formato curricular organizado principalmente por disciplinas, sendo que metade usava PBL em uma seção ou componente específico de alguma disciplina. Entretanto, 65\% dos currículos estão em processo de renovação ou foram modificados dentre os últimos 2 anos. Houve um aumento no percentual de escolas com cursos interdisciplinares, especialmente nas ciências básicas, mas nenhuma mudança no uso da PBL. Porém, as previsões para um currículo integrado estavam dentre as prioridades. Além disso, o treinamento do corpo docente para melhorar suas habilidades de ensino e métodos de avaliação foi listado como a necessidade mais fundamental para apoiar a inovação futura ${ }^{24}$.

$\mathrm{Na}$ China, mesmo com as novas exigências na formação do aluno com uma visão integral, ainda há resistência e dificuldade para alcançar estes objetivos. A Escola de Estomatologia na Universidade de Wuhantem foi pioneira na implementação de inovações, reestruturando o currículo através da introdução do PBL e outras estratégias na formação clínica. Apesar de a PBL estar em seus estágios iniciais, há um entusiasmo com esta abordagem em termos cognitivos, afetivos e habilidades psicomotoras, tanto do corpo docente quanto dos alunos ${ }^{17}$.

Diferentemente da medicina, ao invés de programas completos do PBL, muitos cursos na Odontologia têm apresentado seu currículo híbrido ${ }^{18,31}$. Um currículo híbrido consiste na mescla de sessões de PBL, palestras, aulas laboratoriais, tutoriais, módulos on-line e prática clínica. Isso proporciona ao aluno diferentes estímulos de aprendizagem $^{13}$.

Este é o caso da Universidade de Adelaide, a primeira escola de Odontologia na Austrália a adotar a metodologia PBL. Seu currículo está estruturado em blocos temáticos, não em disciplinas (integração horizontal), assim como são integrados ao longo dos cincos anos de curso (integração vertical $)^{18}$. Não só adotam sessões do PBL, mas também outros estímulos de aprendizagem, tais como: palestras, aulas laboratoriais e clínicas. O perfil também foi adotado na Faculdade Ostrow de Odontologia da Universidade do Sul da Califórnia (OSDUSC), em que a pedagogia PBL é utilizada ao longo dos dois primeiros anos. Após, há uma combinação de módulos tradicionais, pré-clínicos e clínicos ${ }^{31}$.

\section{Sucessos e desafios}

Os alunos têm respondido favoravelmente a este tipo de currículo (PBL), pois permite a aprendizagem situacional, o desenvolvimento de competências em clima de motivação, participação ativa e alto nível de satisfação dos alunos ${ }^{30}$.

A Faculdade de Cirurgia Dentária em Dharan, Nepal, fez um estudo a fim de avaliar o impacto do PBL e os desafios encontrados na sua implantação. Os temas integrados foram articulação temporo-mandibular, músculos da mastigação e oclusão, perpassando por várias disciplinas do currículo tradicional. Os alunos tiveram um período de 5 dias (40 horas) de PBL, com seus tutores previamente treinados. Após a conclusão do módulo, a maioria dos alunos que participaram da pesquisa preferiu o ensino 
em PBL por ser mais interativo e colaborativo, sendo que a integração de disciplinas lhes forneceu perspectivas diferentes para a mesma doença ${ }^{34}$.

Alunos da Universidade de Malmö foram avaliados quanto a sua educação e satisfação profissional. Egressos entre 1995 e 1999 foram questionados sobre aspectos de sua educação, desempenho profissional e interesse em pós-graduação. A maioria dos entrevistados se declarou preparado para o exercício profissional e se disseram satisfeitos quanto à sua profissão, afirmando que o ensino em PBL foi um ponto valioso na sua formação. Além disso, $77 \%$ dos entrevistados manifestaram interesse em especializações ${ }^{33}$. Apesar da resposta positiva à metodologia PBL, os alunos apontam a falta de palestras como ponto negativo do método, o que denota certa relutância dos alunos em se tornarem aprendizes independentes.

Outra crítica comum à PBL é a presença de lacunas no conhecimento, já que o aluno não possui disciplinas delimitando os assuntos. E isto é dependente da habilidade do facilitador, conhecimento prévio do aluno e qualidade dos casos. Antes de abordar este assunto, deve-se saber que também há lacunas presentes no currículo tradicional, uma vez que não são todos os alunos que possuem $100 \%$ de aproveitamento.

No processo da PBL, um dos primeiros passos é identificar os problemas presentes no caso ${ }^{13}$. Com base nos problemas, os próximos passos envolvem engajar os alunos ao pensamento crítico, para definir as suas ideias e hipóteses que explicam os fatos. Essas são discutidas entre os alunos, a fim de encontrar as lacunas no conhecimento e determinar as necessidades de aprendizagem para confirmar suas idéias/hipóteses. Assim, apesar de haver objetivos de aprendizagem previstos pelo corpo docente, estes podem não ser atingidos, dependendo do rumo que os alunos tomarem. O estudo Hagparast et al. ${ }^{25}$ comparou as necessidades de aprendizagem geradas por alunos de quatro grupos de PBL de um programa de doutorado de Cirurgia Dentária. Destes, apenas um grupo apresentou diferenças estatisticamente significantes de aprendizagem. Para resolver este problema, o tutor pode usar questões norteadoras para facilitar as investigações do grupo de conceitos importantes.

Então, para converter um currículo educacional de Odontologia tradicional para PBL é fundamental o desenvolvimento do corpo docente, ou seja, o professor deve estar apto para ser um tutor, ao delinear os objetivos de cada caso clínico apresentado ${ }^{15}$. O corpo docente deve estar preparado para que a abordagem de ensino não seja mais centrada do professor, e sim no aluno. Um exemplo é a escola de Wuhan na China, a qual formou seu corpo docente em PBL, sendo que apenas os professores certificados estavam aptos a serem tutores ${ }^{17}$, otimizando a aplicação do método.

Ao se detectar problemas, não só no corpo docente, mas também na busca do sucesso da metodologia, é necessária avaliação constante e dinamismo. No estudo da Universidade de British, Columbia, foi realizada a avaliação do currículo, a partir da percepção dos alunos. Embora a PBL funcionasse no curso de Odontologia, foram identificados alguns pontos para a melhoria do curso: os alunos se queixaram da discrepância de comentários entre os diferentes tutores, da falta de feedback e dos relatórios exigidos como complemento, considerados extensos e cansativos. Com estes resultados, houve adaptações no currículo para melhorar o desempenho dos alunos $^{22}$, no intuito de 
otimizar a aprendizagem do aluno e alcançar os objetivos propostos.

Por fim, deve-se ter cautela quando houver a integração de cursos, fato que ocorre principalmente durante o aprendizado das ciências básicas. Um estudo avaliou o impacto da integração dos cursos de Medicina e Odontologia na aprendizagem dos alunos, 279 alunos de Medicina e 100 alunos de Odontologia estudaram juntos, utilizando o método PBL durante os dois primeiros anos da faculdade. No final da avaliação, os alunos de Odontologia relataram que se sentiam excluídos, pois eles tinham que se deslocar até a área da medicina e não tinham acesso a todos os espaços, prejudicando o processo de aprendizagem. Com isso, percebe-se que os fatores contextuais devem ser considerados e estudados, pois podem impactar negativamente em experiências de aprendizagem dos alunos ${ }^{19}$.

\section{Ensino Tradicional versus Aprendizagem Baseada em Problemas}

Estudos na literatura odontológica avaliam a eficácia da PBL comparada ao ensino tradicional. Uma revisão sistemática da literatura realizada em $2014^{8}$ mostrou a necessidade de estudos bem delineados, pois ainda há um número muito limitado de estudos controlados bem desenhados que abordem este tema. Os resultados encontrados foram que a metodologia PBL aumenta o conhecimento/desempenho do aluno para as situações clínicas, promove a autopercepção quanto ao preparo para a vida profissional e influencia na sua confiança na prática clínica. Outra revisão sistemática sobre o ensino Odontológico em PBL na China encontrou um efeito positivo na teoria e prática dos alunos, mostrando a metodologia PBL como superior ao ensino tradicional ${ }^{28}$.
Para avaliar as diferenças de aprendizagem entre o ensino tradicional e a PBL, foi realizado um estudo com alunos e professores da Universidade Europea de Madrid, cujos 2 grupos de alunos receberam diferentes tipos de ensino sobre cuidados especiais em Odontologia: um grupo assistiu a aulas expositivas e o outro frequentou aulas em PBL. Apesar dos resultados de aprendizagem avaliados serem ligeiramente maiores em alunos PBL, não houve diferenças estatisticamente significativas entre os grupos $^{32}$.

Outros estudos ${ }^{21,27}$ também não encontraram diferenças na aprendizagem ao longo da vida entre alunos que receberam o ensino tradicional e a PBL. No estudo de Polyzois et al. ${ }^{21}$ ambos os grupos tiveram uma proporção semelhante de ingresso em pósgraduações. Houve uma tendência maior de assinaturas em periódicos odontológico entre alunos que foram formados em faculdades com um currículo tradicional ou híbrido $(35,42 \%)$ do que aqueles que se formaram a partir do currículo PBL $(29,17 \%)$. Com isso, pode-se concluir que a PBL não influenciou na melhoria da aprendizagem ao longo da vida, variáveis demográficas e profissionais parecem ser mais importantes do que o tipo de currículo $^{21}$. Resultados semelhantes foram encontrados por Yiu et al. ${ }^{26}$, sem diferenças no conhecimento clínico entre os alunos formados na Universidade de Hong Kong com um currículo tradicional (1997-2001) e com PBL (2004-2008).

Outro estudo avaliou o desempenho dos alunos de pós-doutorado na Escola de medicina dental de Harvard entre diplomados com a PBL e não (co-residentes). No geral, graduados em PBL se classificaram mais altamente do que os não graduados em todas as competências avaliadas. Porém, não houve 
diferença significativa entre conhecimentos gerais em Odontologia, conhecimentos específicos da especialidade, habilidades préclínicas, habilidades clínicas, a comunicação com a equipe e educação do paciente. Houve diferenças significativas para a comunicação com os pacientes, o pensamento crítico, a aprendizagem independente, o desempenho em pequenos grupos, auto-avaliação e trabalho em equipe. $\mathrm{O}$ desempenho dos diplomados em PBL durante a sua formação de pós-doutorado correspondeu às expectativas e foram semelhantes aos nãograduados para competências tradicionais do programa de residência. No entanto, a formação PBL parece fornecer aos graduados habilidades aprimoradas em aprendizagem independente, comunicação e habilidades de cooperação ${ }^{16}$.

Em um estudo que avaliou a aprendizagem de alunos sobre o papel do profissional e a ética, através de situações clínicas (PBL), os alunos tiveram seu senso de responsabilidade como dentista melhorados, quando comparados ao ensino tradicional ${ }^{20}$.

\section{DISCUSSÃO}

Avanços na metodologia de ensino na Odontologia são necessários, para promover metodologias ativas, direcionadas às necessidades de aprendizagem do aluno, facilitando o desenvolvimento de habilidades, como a de solucionar problemas e de aprendizagem contínua. Dentre essas metodologias, está a PBL, em que há a integração das ciências básicas e clínicas, extinguindo-se as disciplinas. A integração tem como objetivo eliminar a redundância no currículo e formar um profissional com uma visão integral do conhecimento, sem recortes $^{29}$.

O professor teria papel de facilitador, auxiliando o aluno a explorar o problema, para assim extrair os fatos relevantes e gerar hipóteses. Além disso, ele deve identificar as necessidades de aprendizagem dos alunos ${ }^{15,17}$. Este novo papel exigirá conhecimento prévio, sensibilização e prática, sendo um dos primeiros desafios do professor no emprego desta metodologia ${ }^{15,17}$.

É importante ressaltar que, ainda são necessários estudos da PBL em Odontologia, pois há um número limitado de publicações e grande parte destas são da área da medicina ${ }^{10}$, ou baseada na literatura médica. E isto se torna um problema, pois as competências necessárias e o foco da educação para a formação de alunos de Medicina e de Odontologia são diferentes. Então, extrapolar resultados encontrados em estudos da educação médica para a Odontologia pode ser limitado ${ }^{8}$.

Ao analisar os estudos e revisões de literatura, conclui-se que há uma necessidade de estudos controlados, bem desenhados na educação odontológica sobre a eficácia da PBL especialmente a nível curricular com a finalidade de promover a qualidade da evidência científica. Uma vez que, a maioria dos estudos foram classificados como sendo de fraca qualidade e com baixa quantidade de sujeitos de pesquisa ${ }^{8,28}$.

Apesar disto, a evidência desta análise sugere que a PBL não afeta negativamente o conhecimento dos alunos. E apesar de contradições $^{8,17,26-28}$, pode haver melhoria no desenvolvimento das competências dos alunos que aprenderam com a PBL, quando comparados com o ensino tradicional. Além disso, os alunos se sentem mais preparados para a vida profissional. Isto pode ser relacionado à rotina de aprendizagem dos alunos com a PBL, pois os mesmos vivenciam a resolução de problemas desde o início de sua 
formação ${ }^{16,}{ }^{20}$. Não houve diferença na educação permanente entre os estudos, pois os alunos buscavam igualmente cursos de pósgraduação. Este perfil geralmente é dos alunos que se destacam, fator independente da metodologia de aprendizagem ${ }^{21}$.

A problemática de debate quanto ao uso ou não da PBL não deve ser definida apenas como ensino centrado no aluno, ou no professor. Deve-se levar em consideração o conhecimento gerado, através de discussão, pesquisa e até mesmo palestras dirigidas por professores. $\mathrm{O}$ artigo de Tonwsend et al. ${ }^{35}$ (2012) pressupõe que há momentos em que todas estas concepções podem ser valiosas em educação odontológica; uma etapa para a transmissão de conhecimentos e outra em que os alunos precisam para se envolver ativamente no processo de diagnosticar e compreender problemas. Uma palestra sem sentido não é melhor ou pior do que uma classe PBL sem sentido. A PBL não deve ser vista como única opção para promover o processo ensino-aprendizagem. Também existem muitas outras metodologias ativas que podem ser eficientes na educação Odontológica ${ }^{11}$.

Como observado neste artigo, a PBL tem-se mostrado uma ferramenta promissora na educação da Odontologia ${ }^{29}$. Sendo que, currículos híbridos planejados e integrados são colocados como os mais apropriados na Odontologia $^{13}$. Em que são caracterizados como uma mescla da PBL, com palestras, aulas laboratoriais, tutoriais, módulos on-line e prática clínica, proporcionando ao aluno diferentes estímulos de aprendizagem ${ }^{13}, 18,31$.

\section{CONCLUSÃO}

A metodologia PBL se apresenta como uma alternativa no campo da educação em Odontologia. É considerada uma ferramenta de trabalho para professores, que ao utilizá-la promovem nos alunos capacidades cognitivas. Estas contribuem para o desenvolvimento do raciocínio clínico integrado, com perfil direcionado à educação permanente e habilidades cognitivas de diagnosticar e resolver o problema alicerçado no processo de ensino-aprendizagem constante. Mais estudos no ensino da Odontologia devem ser realizados, bem delineados e com amostras suficientes, para mostrar os efeitos da metodologia PBL e encontrar pontos que necessitam melhorias.

\section{ABSTRACT \\ Problem-Based Learning in Dentistry: a critical literature review}

Contemporary society imposes constant challenges in the areas of health and education, founded on permanent revisions and redesigns for training. This article promotes a critical review of the literature on problem-based learning (PBL) directed to the teaching of Dentistry. Data bases consulted were Medline (via EBSCO), SCIELO, BBO and LILACS (BVS). The keywords have been defined based on previously selected articles. The PBL is presented as an alternative methodology for training and education in Dentistry. It isconsidered a working tool for teachers seeking to develop in their students cognitive skills promoting the development of integrated clinical reasoning with the continuing education directed profile.

Descriptors: Problem-Based Learning. Dentistry. Teaching.

\section{REFERÊNCIAS}

1. Brasil. Ministério da Educação. Conselho Nacional de Educação. Câmara de Educação Superior. Resolução CNE/CES, 19 de fevereiro de 2002. Institui as Diretrizes Curriculares Nacionais do Curso de Graduação em Odontologia. Brasília, 2002. Disponível 
em: http://portal.mec.gov.br/cne/arquiv os/ pdf/CES032002.pdf

2. Toassi RFC, Stobäus CD, Mosquera JJM, Moysés SJ, TOASSI R. Currículo integrado no ensino de Odontologia. Interface-Comunic Saúde Educ. 2012;16 (41):529-42.

3. Albuquerque VS, Batista RS, Tanji S, Moço ET-SM, Albuquerque V. Currículos disciplinares na área de saúde. Interface-Comunic Saúde Educ. 2009;13(31):261-72.

4. Paula L, Bezerra ACB. A estrutura curricular dos cursos de Odontologia no Brasil. Rev ABENO. 2003;3(1):7-14.

5. Bastos CC. Metodologias ativas. 2006. Disponível em: http://educacaoemedici na.blogspot.com.br/ Acesso em: 14 agosto de 2015.

6. Freire P. Pedagogia da autonomia: saberes necessários à prática docente. São Paulo: Paz e Terra. 1996.

7. Berbel NAN. As metodologias ativas e a promoção da autonomia de estudantes. Semin Ciênc Soc Hum. 2012;32(1):2540

8. Bassir SH, Sadr-Eshkevari P, Amirikhorheh S, Karimbux NY. Problem-based learning in dental education: a systematic review of the literature. J Dental Educ. 2014;78(1):98109.

9. Donner RS, Bickley H. Problem-based learning in American medical education: an overview. Bull Med Libr Assoc. 1993;81(3):294.

10. Townsend G, Winning T. Research in PBL - where to from here for dentistry? Eur J Dent Educ. 2011;15(3):193-98.

11. Fincham AG, Shuler CF. The changing face of dental education: the impact of PBL. J Dent Educ. 2001;65(5):406-21.
12. Barrows HS. The essentials of problembased learning. J Dent Educ. 1998; 62(9):630-33.

13. Winning T, Townsend G. Problem-based learning in dental education: what's the evidence for and against...and is it worth the effort? Aust Dent J. 2007;52(1):2-9.

14. Zheng JW, Zhang SY, Yang C, Zhang ZY, Shen GF. Creating an effective PBL case in oral and maxillofacial surgery at a Chinese dental school: a dental education primer. J Dent Educ. 2011; 75(11):1496501.

15. Saunders TR, Dejbakhsh S. Problembased learning in undergraduate dental education: faculty development at the University of Southern California School of Dentistry. J Prosthodont. 2007; 16(5):394-99.

16. Thammasitboon K, Sukotjo C, Howell H, Karimbux N. Problem-based learning at the Harvard School of Dental Medicine: self-assessment of performance in postdoctoral training. $\mathrm{J}$ Dent Educ. 2007;71(8):1080-89.

17. Huang C, Bian Z, Tai B, Fan M, Chiu-Yin K. Dental education in Wuhan, China: challenges and changes. J Dent Educ. 2007;71(2):304-11.

18. Saliba NA, Moimaz SAS, Chiaratto RA, Tiano AVP. A utilização da metodologia PBL em Odontologia: descortinando novas possibilidades ao processo ensinoaprendizagem. Rev Odonto Ciênc. 2008;23(4):392-96.

19. Ajjawi R, Hyde S, Roberts C, Nisbet G. Marginalisation of dental students in a shared medical and dental education programme. Med Educ. 2009;43(3):23845.

20. Ji YA, Kim MK, Lee J. Impacts of problem-based professionalism course in 
dental education. Korean J Med Educ. 2010;22(4):275-81.

21. Polyzois I, Claffey N, Attström R, Kelly A, Mattheos N. The role of the curriculum and other factors in determining the medium-to long-term attitude of the practicing dentist towards life-long learning. Eur J Dent Educ. 2010;14(2):84-91.

22. Whitney EM, Walton JN. Faculty and student perceptions of the success of a hybrid-PBL dental curriculum in achieving curriculum reform benchmarks. J Dent Educ. 2010; 74(12):1327-36.

23. Bearn DR, Chadwick SM. Problem-based learning in postgraduate dental education: a qualitative evaluation of students' experience of an orthodontic problembased postgraduate programme. Eur J Dental Educ. 2010;14(1):26-34.

24. Haden NK, Hendricson WD, Kassebaum DK, Ranney RR, Weinstein G, Anderson EL, et al. Curriculum change in dental education, 2003-09. J Dent Educ. 2010; 74(5):539-57.

25. Haghparast N, Okubo M, Enciso R, Clark GT, Shuler C. Comparing studentgenerated learning needs with faculty objectives in PBL cases in dental education. J Dent Educ. 2011; 75(8):1092-97.

26. Yiu CKY, McGrath C, Bridges S, Corbet EF, Botelho M, Dyson J, et al. Graduates' perceived preparedness for dental practice from PBL and traditional curricula. J Dent Educ. 2011;75(9):127079.

27. Pardamean B. Measuring change in critical thinking skills of dental students educated in a PBL curriculum. J Dent Educ. 2012;76(4):443-53.
28. Huang B, Zheng L, Li C, Li L, Yu H. Effectiveness of problem-based learning in Chinese dental education: a metaanalysis. J Dent Educ. 2013;77(3):37783.

29. Nadershahi NA, Bender DJ, Beck L, Lyon C, Blaseio A. An overview of casebased and problem-based learning methodologies for dental education. J Dent Educ. 2013;77(10):1300-05.

30. Teodoro PC. Enseñanza de la radiología y medicina física en el grado en odontología a través del aprendizaje basado en problemas. FEM (Ed impresa). 2014:221-28.

31. Navazesh M, Rich SK, Tiber A. The rationale for and implementation of learner-centered education: experiences at the Ostrow School of Dentistry of the University of Southern California. J Dent Educ. 2014;78(2):165-80.

32. Moreno-López LA, Somacarrera-Pérez ML, Díaz-Rodríguez MM, CampoTrapero J, Cano-Sánchez J. Problembased learning versus lectures: comparison of academic results and time devoted by teachers in a course on Dentistry in Special Patients. Med Oral Patol Oral Cir Bucal. 2009;14(11):e583e87.

33. Bengmark D, Nilner M, Rohlin $M$. Dentists reflect on their problem-based education and professional satisfaction. Eur J Dent Educ. 2012;16(1):e137-e45.

34. Rimal J, Paudel BH, Shrestha A. Introduction of problem-based learning in undergraduate dentistry program in Nepal. IntJ App Basic Med Res. 2015;5(Suppl 1):S45-S49.

35. Townsend GC, Kim M, Sankey D. Dynamic Systems (Complexity) theory as a new conceptual model for researching 
PBL in dental education. Eur $\mathrm{J}$ Dent Educ. 2012;16(1):43-51.
Correspondência para:

Juliana Schaia Rocha

e-mail: julianaschaia@hotmail.com

Rua Alf. Angelo Sampaio, 2692

80730-460 - Curitiba/PR 